\title{
Radiance Spectroscopy Tool Box for Characterizing Au Nanoparticles in Tissue Mimicking Phantoms as Applied to Prostate
}

\author{
Serge Grabtchak ${ }^{1,2,3 *}$, Tyler J. Palmer ${ }^{1}$ and William M. Whelan ${ }^{1,4}$
}

${ }^{1}$ Department of Physics, University of Prince Edward Island, 550 University Avenue, Charlottetown, PEI C1A4P3, Canada

${ }^{2}$ Department of Electrical and Computer Engineering, Dalhousie University, 1360 Barrington Street, Halifax, NS B3J1Z1, Canada

${ }^{3}$ Department of Physics, Dalhousie University, 6310 Coburg Road, Halifax, NS B3H3J5, Canada

${ }^{4}$ Atlantic Veterinary College, 550 University Avenue, Charlottetown, PEI C1A4P3, Canada

\begin{abstract}
Background: We have developed a new approach to map localized inclusions of gold nanoparticles in the Intralipid-1\% liquid phantom. Our goal was to show that combined spectroscopic and angular snapshots of liquid phantoms and phantoms with inclusions allow obtaining information relevant for prostate cancer diagnostics and treatment.

Methods: A combination of the point radiance spectroscopy and white light spectroscopy was used to measure angular resolved light distribution in 450-900 nm spectral range in Intralipid-1\% liquid phantoms with and without localized inclusions of gold nanoparticles.

Results: Characteristic spectro-angular snapshots of the liquid phantom alone and with the localized inclusion of gold nanoparticles were obtained. For liquid phantoms without inclusions, the snapshots demonstrate wavelength dependent light distribution inside the turbid medium, visualize the transparency window and provide a quantification of angular spread of different wavelengths of light. For liquid phantoms with gold inclusions, the approach allows to isolate the spectroscopic signatures of the inclusions from the background, identify locations of the inclusions in the angular domain and quantify the detection limits in terms of the contrast value attainable for the selected quantity of gold nanoparticles located at the specific depth in tissue. A detection of $3 \times 10^{13}$ particles up to $25 \mathrm{~mm}$ deep in Intralipid-1\% was demonstrated.
\end{abstract}

Conclusions: The encouraging results indicate a promising potential of radiance spectroscopy in prostate treatment and diagnostics with gold nanoparticles.

Keywords: Intralipid; Gold nanoparticles ( $\mathrm{Au} \mathrm{NPs);} \mathrm{Prostate;}$ Radiance; Light scattering; Spectro-angular mapping

\section{Introduction}

Nanotechnology utilizes nanoscale-size materials that offer new attractive properties and is positioned to revolutionize cancer medicine [1]. For prostate applications, the use of gold nanoparticles (Au NPs) in particular, has advanced along four major fronts: 1) interstitial nanoparticle-mediated laser thermal therapy [2], 2) cancer diagnostics via delineation of cancer affected areas with NPs conjugated to monoclonal antibodies [3], 3) enhanced radiation sensitivity and toxicity in prostate cancer cells containing Au NPs [4], 4) brachytherapy implants of radioactive ${ }^{198} \mathrm{Au}$ NPs placed into a prostate gland to deliver the radiopharmaceutical dose directly to the tumor site [5].

Unless NPs are injected directly to the tumor and followed by an immediate application, delivery methods and the time elapsed between the incubation and the application possess a serious problem for establishing NPs presence. Whether one uses functionalized NPs with specific binding to tumor sites or non-functionalized NPs with preferential accumulation at tumor sites, the main challenge is "...the inability to visualize or quantify the global concentration or spatial distribution of these particles within the tumors" [6]. Registering Au NPs in the particular region of the tumor prior application would provide the first encounter with the affected area thus facilitating the following treatment procedure (including optimization of irradiation conditions for thermal therapy). Therefore, for all applications an ability to identify, locate and quantify fixed loads of Au NPs in the prostate gland via non-invasive or minimally invasive methods is highly desirable. Optical-based techniques use a non-ionizing radiation, and light penetration depth can be optimized based on wavelength dependent tissue optical properties. However, in turbid media (i.e., tissues) photons undergo multiple scattering, and exhibit a chaotic random walk, which results in a significant spread in spatial distribution. Hence, information about localized optical properties is difficult to decode from light-tissue data. The ultimate goal of all optical techniques is in developing approaches for extracting the information about local optical properties of turbid media.

Among the techniques that have been successfully employed to address this challenge is Diffused Optical Spectroscopy (DOS) [7]. The technique is based on collecting reflected light and extracting relevant optical properties by coupling measured optical data to a model for diffuse reflectance based on a diffusion approximation. This technique was able to extract the concentration of gold nanoshells in tumors,

*Corresponding author: Serge Grabtchak, Department of Physics, University of Prince Edward Island, 550 University Avenue, Charlottetown, PEI C1A 4P3 Canada Tel: +1-902-566-6078; Fax: +1-902-566-0483; E-mail: sgrabtchak@upei.ca

Received October 24, 2011; Accepted November 28, 2011; Published November 30, 2011

Citation: Grabtchak S, Palmer TJ, Whelan WM (2011) Radiance Spectroscopy Tool Box for Characterizing Au Nanoparticles in Tissue Mimicking Phantoms as Applied to Prostate. J Cancer Sci Ther S1. doi:10.4172/1948-5956.S1-008

Copyright: (c) 2011 Grabtchak S, et al. This is an open-access article distributed under the terms of the Creative Commons Attribution License, which permits unrestricted use, distribution, and reproduction in any medium, provided the original author and source are credited. 
but it cannot provide any spatial information about their location [7]. Another technique, narrow band infrared imaging can visualize Au nanoshell distribution but only in superficial tissue layers $(\sim 1 \mathrm{~mm}$ depth) [8].

Point radiance spectroscopy is an optical technique whereby light is collected within a small, well-defined angular cone thus providing information on angular light distribution at a selected point in turbid medium. Interestingly, the radiance-based light dosimetry was first applied to prostate interrogations [9]. A later work from the same group was devoted to optimization of modeling algorithms for extracting global optical properties of phantoms from radiance measurements [10]. Building on this we have demonstrated a potential for radiancebased tissue coagulation detection [11] and optical property determination [12]; and more recently, radiance-based detection of localized Au NPs inclusions inside Intralipid phantoms [13,14].

The scattering properties of the Intralipid-1\% are similar to those of human prostate. The absorption coefficient of Intralipid- $1 \%$ in the VIS-NIR range is from 0.01 to $0.1 \mathrm{~cm}^{-1}$. This is somewhat lower than the absorption coefficient of human prostate which exhibits significant inter-organ and intra-organ variability that translates to a broad range of $0.1-1.6 \mathrm{~cm}^{-1}$ as measured at $732 \mathrm{~nm}$ in prostate carcinoma [14]. It is well known that it is the highly scattering nature of biological tissues that is responsible for loosing optical resolution and increasing blurring of optical images with depth. Hence, it was our intention to isolate scattering from absorption of the prostate and focus mostly on scattering for the phantom studies. We performed measurements within a $5 \mathrm{~cm}$ area to mimic scattering over a prostate of average dimension. Once the developed methodology is applied to prostate studies, new detectability limits in terms of the minimal concentration and detection distance can be established because of the different absorption coefficient of prostate. However, the spectro-angular approach would remain valid.

In this work, a new approach to detecting a specific payload of $\mathrm{Au}$ NPs in turbid media based on a combined spectral and optical data set is presented. The experimental data are acquired in a tissue equivalent liquid phantom with scattering properties similar to human prostate. We obtained a complete spectro-angular characterization of the phantom material (Intralipid-1\%), visualized experimentally the biomedical transparency window and its evolution with distance and mapped localized inclusions of Au NPs in Intralipid-1\% simultaneously in the spectroscopic and angular domains. The ease of manipulation with the source, the detector and the Au NPs sample inside the liquid phantom allowed targeting sample's placement geometries relevant for the actual prostate.

\section{Materials and Methods}

\section{Intralipid phantom and gold nanoparticles}

Intralipid-1\% served as a background optical medium. It was prepared by a volume dilution of Intralipid-20\% (Sigma-Aldrich, Oakville, ON, Canada) stock solution. Water-based 5-nm diameter Au colloid with a stock concentration of $5 \times 10^{13}$ particles $/ \mathrm{mL}$ (Ted Pella, Redding, CA, USA) filled a $3.5-\mathrm{mm}$ diameter quartz capillary with a volume $\sim 0.7 \mathrm{~mL}$ that was completely immersed into the Intralipid solution. The immersion length was $\sim 6 \mathrm{~cm}$. Therefore, the estimated maximum number of Au nanoparticles that were detected was $\sim 3 \times 10^{13}$. The extinction coefficient of $5-\mathrm{nm}$ Au nanoparticles is dominated by the absorption due the localized plasmon resonance at a wavelength of $\sim 520 \mathrm{~nm}$ as shown on Figure 1 .

\section{Instruments}

A detailed description of the experimental setup for radiance measurements has been published elsewhere $[13,14]$. Therefore, only essential details are given here. Figure 2 shows the experimental geometry (cross-section) that consists of a source fiber, a detection fiber and an Au NPs filled tube (i.e., the target). A blackened, $18 \mathrm{~cm}$ sided Lucite box was filled with Intralipid-1\%. A tungsten halogen white light source $(20 \mathrm{~W})$ was connected to a fiber terminated with an isotropic spherical diffuser. The total power output from the spherical diffuser was estimated to be $\sim 18 \mathrm{~mW}$. A side firing fiber (Polymicro Technologies, Phoenix, AZ, USA) with a well-defined angular acceptance window ( $\sim 10$ degrees in water) served as a radiance detector. The side firing fiber was mounted on a computer-controlled rotation stage ensuring a full 360-degree rotation with a 2-degree step. The output of the radiance detector was connected to spectrometer (USB 4000, Ocean Optics, Dunedin, FL, USA) for spectral acquisition. Signal averaging was based on four measurements at each angular step requiring approximately 10

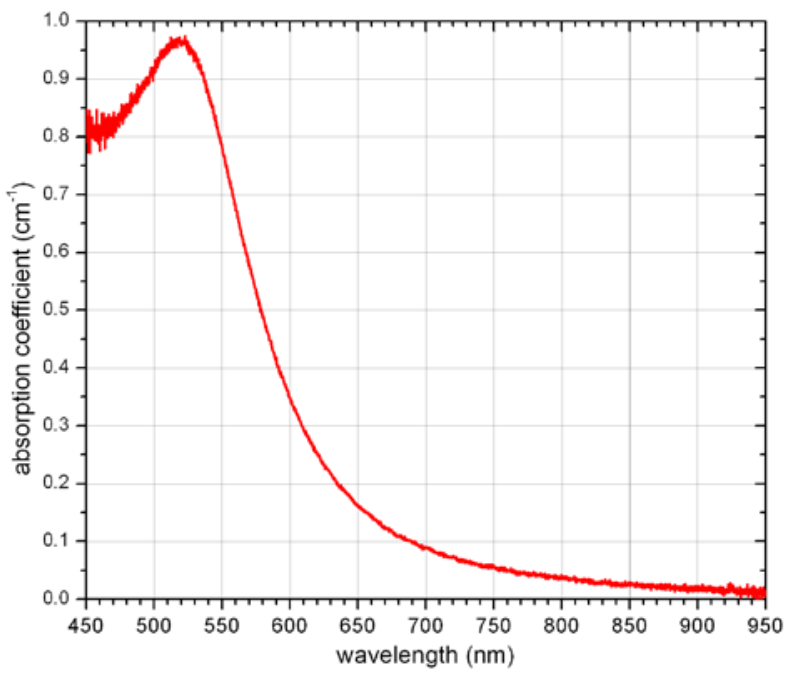

Figure 1: Experimental extinction spectrum of a colloidal solution of 5-nm gold nanoparticles.

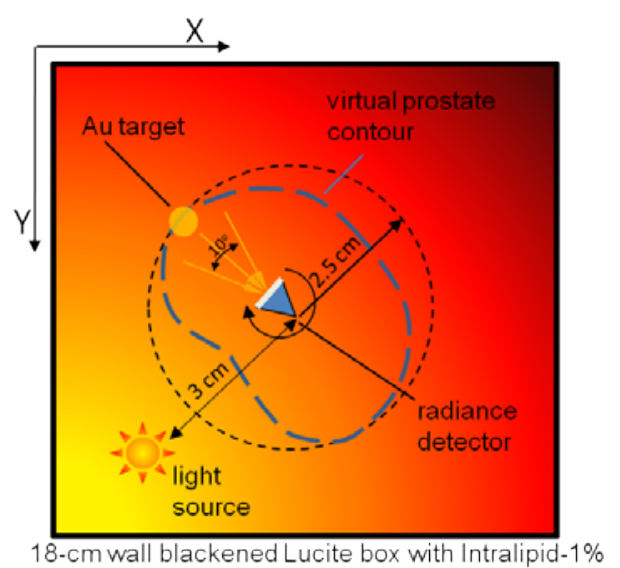

Figure 2: 2D cross-section of the measurement box showing relative arrangement of light source, radiance detector and gold sample. Dotted line outlines a contour of a virtual prostate. 
minutes to acquire a single complete angular profile. Positions of both fibers and the tube were independently controlled in the $\mathrm{x}, \mathrm{y}$-plane by three translation stages with a sub-mm positioning accuracy.

The positioning of the fibers/tube in the Intralipid-1\% phantom was chosen to mimic a prostate application in which the radiance detector is placed in the urethra, the diagnostic white light source is placed in the rectum and the Au NPs target is located at a peripheral zone of the prostate (Figure 2). This represents an idealized, minimally invasive approach to radiance-based detection in prostate, but at the same time the separations between the radiance detector and the Au NPs target $(\sim 2.5 \mathrm{~cm})$ and between the white light source and the detector $(\sim 3 \mathrm{~cm})$ correspond to worst case scenario in terms of target detectability due to limited optical penetration in tissues.

\section{Results and Discussion}

Spectral and angular information is presented as a spectro-angular map, hence obtaining a snapshot of the medium under selected experimental conditions. Spectral profiles measured at every angular step in Intralipid-1\% were assembled into the Intralipid matrix where columns corresponded to angles from 0 to 360 degree and rows were wavelengths from $450 \mathrm{~nm}$ to $950 \mathrm{~nm}$. To exclude spectral variations due to the white light source and the detector, all radiance data obtained in the Intralipid-1\% were divided by corresponding values in air. This ratio of the Intralipid matrix to the air matrix represents a relative radiance that reflects properties of Intralipid-1\% only. This normalization approach is similar to standard spectroscopic measurements using a blank target. An example of the contour plot of the relative radiance in Intralipid-1\% obtained at $16.5 \mathrm{~mm}$ sourcedetector separation is shown on Figure 3. Same color and contour lines correspond to regions of equal intensity. Areas of highest transmission are more red while areas of lowest transmission are blue. The plot is essentially a snapshot of the wavelength-selective angular distribution of light in Intralipid-1\% at the chosen distance. It is easy to show that viewing the plot along the vertical direction at the fixed angle gives the transmission spectrum of Intralipid-1\% at this angle (see the inset corresponding to $a-a$ line in Figure 3). Similar, dissecting the plot horizontally provides the angular distribution of light for the selected wavelength in Intralipid (see the inset corresponding to $b-b$ line in

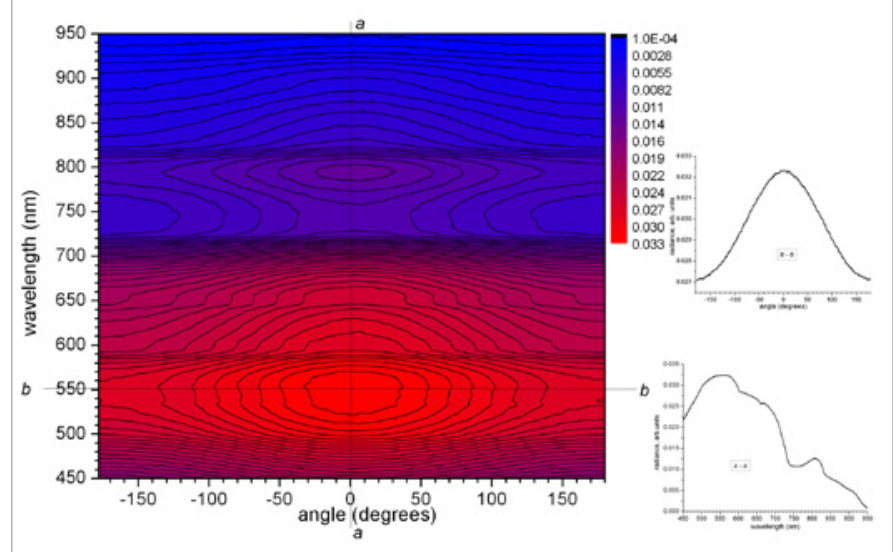

Figure 3: Spectro-angular map of Intralipid-1\% obtained at $16.5 \mathrm{~mm}$ sourcedetector separation distance (no gold sample). Inset a-a: cross-section along the vertical line as indicated. Inset b-b: cross-section along the horizontal line as indicated.

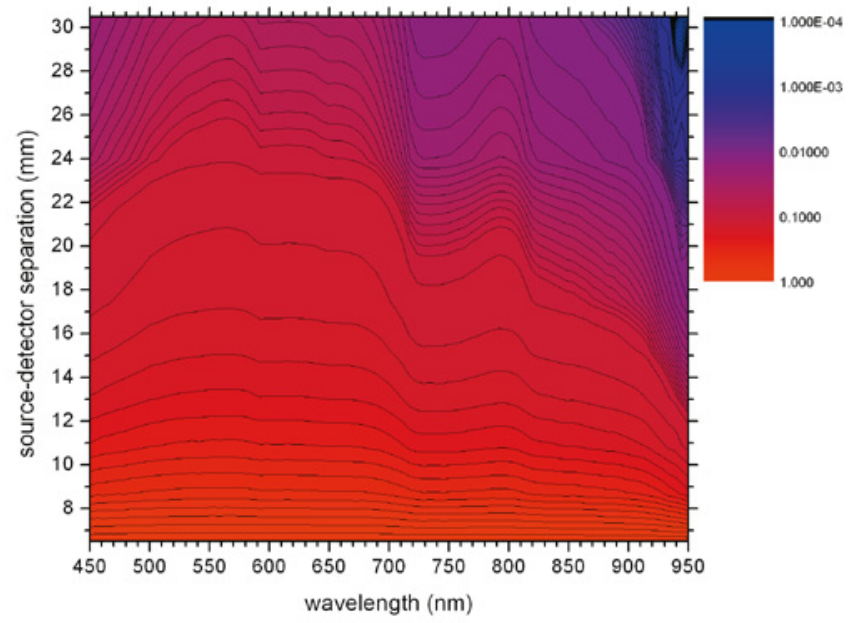

Figure 4: Spectral variation of radiance with distance along 0-degree angle in Intralipid-1\%. Note a logarithmic scale for radiance values.

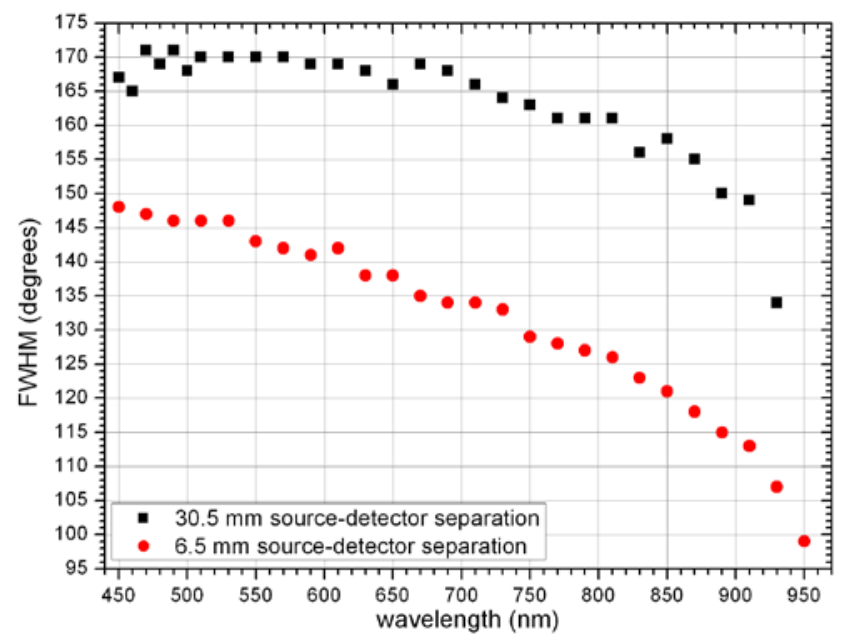

Figure 5: Variation of the full-width half-maximum (FWHM) of angular distribution of light vs. wavelength in Intralipid- $1 \%$ for $6.5 \mathrm{~mm}$ and $30.5 \mathrm{~mm}$ sourcedetector separations.

Figure 3). Notice a substantial (relative to the maximum) value of the backscattered light (at 180 degrees). The amount of the backscattered light increases with increasing the source-detector separation because it increases a probability of reversing a path for photons after successive multiple scattering events [9]. A 2D analysis of the contour plot provides the wavelength and the angle of the maximum transmission, i.e. $\sim 550 \mathrm{~nm}$ and 0 degree, correspondingly. This is not surprising because Intralipid-1\% is known to be a homogeneous isotropic medium with preferential forward scattering properties [14] and therefore, the maximum of the transmitted signal will be observed when the detector is facing the light source (i.e. at 0 degrees). Notice the water absorption feature that is delineated as a valley at $\sim 750 \mathrm{~nm}$ on the contour plot shown in Figure 3.

A similar snapshot can be obtained in the prostate by placing the detector into the urethra and illuminating the prostate through the rectal wall. Also, using a standard brachytherapy grid can provide multiple interstitial locations for placement of the illuminating fiber adding 

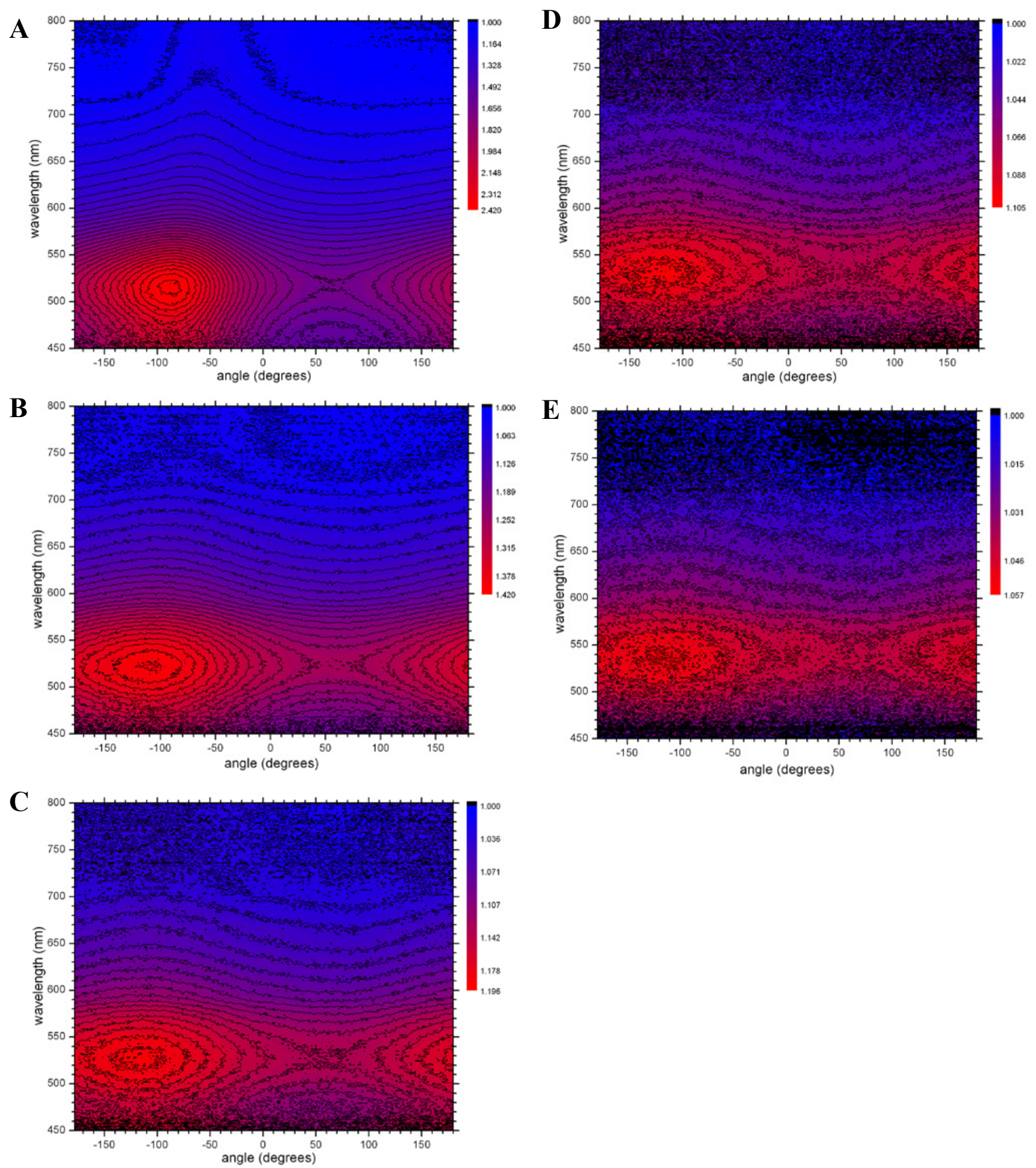

Figure 6: Spectro-angular maps of a localized inclusion of gold nanoparticles in Intralipid-1\% for selected sample-detector separations: a) $5 \mathrm{~mm}$, b) $10 \mathrm{~mm}$, c) $15 \mathrm{~mm}$, d) $20 \mathrm{~mm}$, e) $25 \mathrm{~mm}$.

more flexibility to illumination conditions. The prostate snapshot would carry information about spectral properties of the prostate gland prior to incubating it with Au NPs. The ability to achieve a complete 360 -degree rotation is essential for the technique. While it is relatively easy to obtain it in phantom studies, clinical testing in prostate would require the development of a special catheter/endoscope coupled to a miniaturized rotation stage similar to what has been developed for optical coherence tomography (OCT) applications [15]. 
Obtaining radiance measurements at various source-detector separations provides an opportunity to analyze the combined effects of absorption and scattering on light transmission in phantoms and tissues. When the diffusion approximation is valid, it can be expressed in terms of the well-defined effective attenuation coefficient [16]. The effective attenuation coefficient defines the biomedical transparency window in tissues [17]. We performed distance dependent radiance measurements by varying the source-detector separation from $6.5 \mathrm{~mm}$ (the closest distance achievable with the experimental apparatus) to 30.5 $\mathrm{mm}$ and keeping the detector facing the light source (corresponding to 0 degree). Then all measured values were normalized by the value of radiance measured at the shortest distance, i.e. $6.5 \mathrm{~mm}$. The spectral variation of the radiance with distance is presented on Figure 4 in the form of a spectro-spatial contour plot (note a logarithmic scale for radiance values). One can see that transmission peaks become more sharp and well-defined with distance. With inherent scattering by lipid particles and absorption by two chromophores (Intralipid and water), the wavelength range from $\sim 550 \mathrm{~nm}$ to $\sim 700 \mathrm{~nm}$ appears to be the optimal transmission window for Intralipid-1\%.

Figure 5 demonstrates the effect of wavelength-dependent light scattering in Intralipid-1\% on the full-width half-maximum (FWHM) of angular profiles of light measured at the extremes of source-detector separations, $6.5 \mathrm{~mm}$ and $30.5 \mathrm{~mm}$. The plot was obtained from analyzing sets of angular profiles similar to the one shown at the inset $b-b$ on Figure 3. It is known that multiple scattering in turbid media tends to homogenize light distribution. Knowledge of wavelength-dependent spectral broadening is important when considering intended localized light-tissue interactions like drug illumination in PDT, selective tissue ablation in thermal therapy, optimized light delivery to gold nanoparticles in interstitial thermal therapy or detection of localized optical inhomogeneities in bulk tissues. Results from Figure 5 indicate that even after passing $6.5 \mathrm{~mm}$ distance in Intralipid-1\%, light spreads over $\sim 145$ degree angle for $450 \mathrm{~nm}$ and over 100 degree angle for $950 \mathrm{~nm}$. Increasing the illumination distance up to $30.5 \mathrm{~mm}$ changes the values to $\sim 170$ degrees $(450 \mathrm{~nm})$ and $\sim 135$ degrees $(950 \mathrm{~nm})$. This information may be used when optimizing light delivery to $\mathrm{Au}$ nanoparticles in tissues. A current practice is to use fibers with isotropic cylindrical diffusers for illuminating tissues containing embedded $\mathrm{Au}$ nanoparticles. During a uniform 360-degree illumination, a significant portion of light does not interact with the target (i.e. Au nanoparticles) unless the illuminating fiber is inserted directly in the nanoparticles containing area. Using a side firing fiber as a light source, one can explore target illumination from a distant location that doesn't have to coincide with the target spot. It may result in a better light delivery and optimized power budget depending on the illumination wavelength and distance to the target. A similar optimization can be performed for drug illumination in PDT.

Radiance measurements with the Au NPs tube for target-detector separations of $5 \mathrm{~mm}, 10 \mathrm{~mm}, 15 \mathrm{~mm}, 20 \mathrm{~mm}$ and $25 \mathrm{~mm}$ are shown on Figure 6. The source-detector separation was chosen to be $3 \mathrm{~cm}$ even though in the actual prostate the distance between the prostate and the rectal wall is $\sim 2 \mathrm{~cm}$. The extra distance accounts for the fact that Intralipid-1\% is a weaker absorber than the prostate. Similar to the previous approach, two matrices were constructed. The first was the Intralipid matrix corresponding to the homogenous phantom. The second was the Intralipid+Au matrix corresponding to the phantom with the localized Au inhomogeneity. Since we were interested in absorption rather than transmission properties of the system, we constructed the ratio of Intralipid/(Intralipid+Au). Our blank in this case was pure Intralipid and our sample of interest was Intralipid with the inclusion. The obtained ratio excluded spectral contributions from the white light source, detection system and more important, Intralipid. The only visible features were due to the sample, i.e. colloidal Au. The series of snapshots corresponding to different sampledetector separations are shown on Figure 6. These are raw data that haven't been subject to any post-processing. As one can see, the red area corresponds to the maximum absorption, and it is located in the vicinity of (-90)-degree value (the actual position of the sample). The most distinct changes occur between 5 - and $10-\mathrm{mm}$ separations. The first contour line that is somewhat close to a circle resides firmly at the (-90)-degree mark for 5-mm separation. Shifting sample to the 10-mm location produces two effects: first, the contour lines tend to acquire a more elliptical shape and second, the center of the wouldbe ellipse shifts away from the source towards (-110)-degree mark. All other sample locations demonstrate about the same degree of concentricity of contour lines and an additional 10-degree shift towards (-120)-degree location. It appears that the sample develops some sort of a shadowing effect when seen from a larger distance. We'd like to note that different sample-detector separations correspond to probing the same photon distribution around the sample but at some distance away from the sample. (Changing sample-detector distance produces also some variation in the sample illumination condition by changing the source-sample separation as one can easily envision from Figure 1. However, our additional measurements indicated that the variation in sample illumination doesn't play a major role in the observed effect.) The shadowing effect is a signature of the strong absorber in the highly scattering medium. Because of the ratio approach introduced in the article, we always compare light distribution in the vicinity of the sample with the one in Intralipid with sample absent. Placing the sample inside Intralipid extends the perturbation in light distribution beyond the physical dimensions of the sample. Due to multiple scattering events, photons that cross the sample's area can be found in a large cloud around the sample. Introducing the absorbing sample effectively removes the photons from the cloud they would be normally present and forms the area of the lower photon density that is perceived as the shadow. A remarkable fact is that even with the 30-degree angular uncertainty at larger distances, the angular location of the sample and its spectroscopic signature can be reliably indentified.

By comparing the maximum ("on-sample") and the minimum ("offsample") radiance values along the wavelength of the maximum lightsample interaction (520nm, corresponding to the plasmon resonance), we obtained the contrast value for the specified concentration of $\mathrm{Au}$ colloid in Intralipid-1\%. The contrast value decreased with increasing the sample-detector separation at the fixed angle of 90-degree as follows: $5 \mathrm{~mm}-47 \%, 10 \mathrm{~mm}-11 \%, 15 \mathrm{~mm}-4.8 \%, 20 \mathrm{~mm}-2.1 \%$, $25 \mathrm{~mm}-1.1 \%$. The increase in separation spreads photons over a larger angular range and increases a portion of backscattered light thus leading to lower contrast values.

We were able to obtain the snapshots similar to those shown on Figure 6 for the sample placed at other angular locations. The contrast value changed with the angle for the sample-detector separation fixed at $15 \mathrm{~mm}$ as follows: 0 -degree $-3.6 \%, 45$-degree $-8.2 \%, 90$-degree $4.8 \%, 135$-degree $-4.6 \%, 180$-degree $-2.6 \%$. Notice, that the variation is not as dramatic as for the distance dependent measurements. The numbers reflect a combined effect of a variation in the sample illumination condition and the preferential forward scattering. It bears a practical significance when translated to measurements in the prostate. Illumination via rectum would provide a similar non- 
uniform illumination of the prostate implying that localized inclusions of Au nanoparticles can be detected at any angular location when placing the radiance detector into the urethra. As was discussed earlier [14], the experimental set-up has a $2 \mathrm{D}$ symmetry. For the in-plane measurements, the infinite cylinder represents a localized inhomogeneity. Therefore, in the phantom experiments, Au NPs always occupy a well-defined cylindrical volume. This ideal geometry is needed to evaluate the effect of multiple scattering on target blurring in the plane of detector rotation and to identify signatures of Au NPs in turbid media. However, due to the inherent $3 \mathrm{D}$ nature of scattering, the interaction between light and a capillary with Au NPs can't be confined to in-plane. The actual concentration and distribution of Au NPs in prostate cancer would likely be quite variable and would depend on a number of factors including the tumour vasculature, nanoparticle size and method of delivery. Hence it would be expected that the overall shape of the Au NP distribution would extend nonuniformly in various directions. While the proposed technique can't visualize individual nanoparticles, it can detect localized AuNP concentrations. An implementation of the technique (via a vertical translation of the detecting fiber) for adding a third dimension in locating Au NPs distribution was proposed earlier [14].

$\mathrm{Au}$ NPs detection limits depend strongly on the values of the contrast ratio attainable at the specified depth for the specified density of Au NPs. For the used in this work stock solution of $5 \mathrm{~nm}$ Au NPs, all reported above values correspond to $\sim 3 \times 10^{13}$ particles. In the previous work [14] where different Au NPs were used, the detected volume $\sim 0.7 \mathrm{~mL}$ of $3.8 \times 10^{8}$ particles $/ \mathrm{ml}$ of $250 \mathrm{~nm} \mathrm{Au}$ produced $\sim 3.8 \times 10^{8}$ particles detected at the distance of $6.5 \mathrm{~mm}$ with the contrast value of $3-4 \%$. In a good approximation one can safely ignore the difference in values of the absorption cross-section between $5 \mathrm{~nm}$ and $250 \mathrm{~nm} \mathrm{Au}$ NPs and attribute the detection limits to the concentration only. Our detectability range for Au NPs includes for the example the amount of nanoparticles used in hyperthermia treatment of cancer in mice when $100 \mu \mathrm{L}$ of $2.4 \times 10^{11}$ nanoparticles/mL were injected intravenously [6]. Given a size difference, the payload to the canine and human prostate would exceed this amount. We'd like to stress, that the minimally detectable number of nanoparticles should be always considered in conjunction with a value of contrast ratio achieved at a particular depth in a specific tissue. We are currently working on developing algorithms for extracting the sample-detector distance information and a concentration of Au NPs from the measured data. Once achieved, it would provide a more complete characterization of localized $\mathrm{Au}$ inclusions in the prostate therefore addressing the remaining problems mentioned in the introduction.

Finally, we'd like to comment on the effect of the increased optical absorption in prostate tissues on detectability of Au NPs. It is known from Monte-Carlo modeling and radiance measurements in phantoms with different values of the absorption coefficient, that increasing the background absorption leads to less backscattered signal [9]. The result is a reduction of the overall spread due to scattering thus improving light confinement. We expect that spectro-angular maps of the tissue and of the inhomogeneity in the tissue (Figure 3 and Figure 6) will exhibit less angular spread in the actual prostate facilitating angular detection of the target. At the same time, a total detected signal will be reduced due to additional absorption of prostate that would require revisiting the power budget and establishing new conditions for detectability. This most likely would lower the contrast ratio values, decrease the detection depth and increase the minimal detectable concentration. However, to some degree the changes can be compensated by appropriate adjustments in acquisition parameters (e.g. integration time and number of averages). Also, the biomedical transparency window of the prostate differs from that of Intralipid because of the presence of additional chromophores, hemoglobin in particular. In the current article, the choice of 5-nm Au NPs that absorb at $525 \mathrm{~nm}$ was dictated by a need to match the wavelength of plasmon absorption to the transparency window in the turbid medium under study, Intralipid- $1 \%$ from $\sim 550 \mathrm{~nm}$ to $\sim 700 \mathrm{~nm}$. The same matching requirement would call for using different $\mathrm{Au}$ NPs with plasmon resonance in the biomedical transparency window from $\sim 750 \mathrm{~nm}$ to $\sim 1000 \mathrm{~nm}$ of the prostate.

In conclusion, we presented very encouraging results on detecting spectral and angular signatures of localized inclusions of Au NPs within $6.5 \mathrm{~mm}-30.5 \mathrm{~mm}$ distance from the radiance detector in Intralipid-1\%. The spectro-angular mapping approach described here can be extended to prostate applications.

\section{Acknowledgments}

The authors acknowledge financial support from Natural Sciences and Engineering Research Council, Canadian Institutes of Health Research, Atlantic Innovation Fund and Canada Research Chair program (W. M. Whelan)

\section{References}

1. Nie SM, Xing Y, Kim GJ, Simons JW (2007) Nanotechnology applications in cancer. Annu Rev Biomed Eng 257-288.

2. Schwartz JA, Price RE, Gill-Sharp KL, Sang KL, Khorchani J, et al. (2011) Selective Nanoparticle-Directed Ablation of the Canine Prostate. Lasers Surg Med 43: 213-220.

3. Sokolov K, Follen M, Aaron J, Pavlova I, Malpica A, et al. (2003) Real-time vital optical imaging of precancer using anti-epidermal growth factor receptor antibodies conjugated to gold nanoparticles. Cancer Res 63: 1999-2004.

4. Zhang XJ, Xing JZ, Chen J, Ko L, Amanie J, et al. (2008) Enhanced radiation sensitivity in prostate cancer by gold-nanoparticles. Clin Invest Med 31 E160-E7.

5. Chanda N, Kan P, Watkinson LD, Shukla R, Zambre A, et al. (2010) Radioactive gold nanoparticles in cancer therapy: therapeutic efficacy studies of GA-198AuNP nanoconstruct in prostate tumor-bearing mice. Nanomedicine 6: 201-209.

6. Krishnan S, Diagaradjane P, Cho SH (2010) Nanoparticle-mediated therma therapy: Evolving strategies for prostate cancer therapy. Int J Hyperthermia 26 : 775-789.

7. Zaman RT, Diagaradjane P, Wang JC, Schwartz J, Rajaram N, et al. (2007) In vivo detection of gold nanoshells in tumors using diffuse optical spectroscopy. IEEE J Sel Top Quantum Electron 13:1715-1720.

8. Puvanakrishnan P, Park J, Diagaradjane P, Schwartz JA, Coleman CL, et al (2009) Near-infrared narrow-band imaging of gold/silica nanoshells in tumors. J Biomed Opt 14: 024044.

9. Barajas O, Ballangrud AM, Miller GG, Moore RB, Tulip J (1997) Monte Carlo modelling of angular radiance in tissue phantoms and human prostate: PDT light dosimetry. Phys Med Biol 42:1675-1687.

10. Dickey DJ, Moore RB, Rayner DC, Tulip J (2001) Light dosimetry using the P3 approximation. Phys Med Biol 46: 2359-2370.

11. Chin L, Pop M, Whelan W, Sherar M, Vitkin A (2003) Optical method using fluence or radiance measurements to monitor thermal therapy. Rev Sci Instrs 74: 393-395.

12. Chin LCL, Whelan WM, Vitkin IA (2006) Information content of point radiance measurements in turbid media: implications for interstitial optical property quantification. Appl Opt 45: 2101-2114. 
Citation: Grabtchak S, Palmer TJ, Whelan WM (2011) Radiance Spectroscopy Tool Box for Characterizing Au Nanoparticles in Tissue Mimicking Phantoms as Applied to Prostate. J Cancer Sci Ther S1. doi:10.4172/1948-5956.S1-008

Page 7 of 7

13. Grabtchak S, Palmer TJ, Whelan WM (2011) Spatially-resolved probing of biological phantoms by point-radiance spectroscopy. Israel, SPIE, 78940A.

14. Grabtchak S, Palmer TJ, Whelan WM (2011) Detection of localized inclusions of gold nanoparticles in Intralipid-1\% by point-radiance spectroscopy. J Biomed Opt 16: 077003.

15. Mueller-Lisse UL, Meissner OA, Babaryka G, Bauer M, Eibel R, et al. (2006) Catheter-based intraluminal optical coherence tomography (OCT) of the ureter: ex-vivo correlation with histology in porcine specimens. Eur Radiol 16: 2259 2264.

16. Martelli F, Bassani M, Alianelli L, Zangheri L, Zaccanti G (2000) Accuracy of the diffusion equation to describe photon migration through an infinite medium: numerical and experimental investigation. Phys Med Biol 45: 1359-1373.

17. Weissleder R (2001) A clearer vision for in vivo imaging. Nat Biotechnol 19 316-317.
This article was originally published in a special issue, Prostate Cancer handled by Editor(s). Dr. Gary Guishan Xiao, Creighton University, USA; Dr. Sreenivasa R. Chinni, Wayne State University, USA 